\title{
Development of Tobacco Mosaic Virus Infection Sites in Nicotiana benthamiana
}

\author{
Judit Szécsi, ${ }^{1}$ Xin Shun Ding, ${ }^{2}$ Chae Oh Lim, ${ }^{1,3}$ Mohammed Bendahmane, ${ }^{1}$ Moo Je Cho, ${ }^{3}$ \\ Richard S. Nelson, ${ }^{2}$ and Roger N. Beachy ${ }^{1}$ \\ ${ }^{1}$ Division of Plant Biology, Department of Cell Biology, The Scripps Research Institute, 10550 North Torrey \\ Pines Road, La Jolla, CA 92037, U.S.A.; ${ }^{2}$ The Samuel Roberts Noble Foundation, Plant Biology Division, \\ P.O. Box 2180, Ardmore, OK 73402, U.S.A.; ${ }^{3}$ Plant Molecular Biology and Biotechnology Research \\ Center, Department of Biochemistry, Gyeongsang National University, Chinju 660-701, Korea \\ Accepted 15 October 1998.
}

\begin{abstract}
To monitor infection of Nicotiana benthamiana by tobacco mosaic virus (TMV), leaves were inoculated with viral constructs expressing the green fluorescent protein (GFP) from jellyfish (Aequorea victoria) fused to the movement protein (MP) of TMV (MP:GFP) or as a free GFP in place of the coat protein $(\mathrm{CP})$. Infection sites produced by TMV expressing the MP:GFP appeared as fluorescent rings larger in diameter and less fluorescent than fluorescent disks induced by constructs encoding free GFP. These results suggest that protein expression driven by the MP subgenomic promoter (sgp) initiates and ends earlier and is at lower level than that observed for proteins driven by the CP sgp. Similarly, analyses of cross sections through the infection sites revealed that in different cell types the accumulation of MP:GFP was regulated differently than the accumulation of free GFP. Immunocytochemistry and electron microscopy showed that near the leading edge of the fluorescent ring the MP:GFP and the viral $126 \mathrm{kDa}$ and $183 \mathrm{kDa}$ replicase proteins accumulated in paired cytoplasmic bodies that formed often on opposite sides of adjacent cell walls containing plasmodesmata. In the dimly fluorescent center of the rings the $126 \mathrm{kDa}$ and 183 kDa proteins, but not the MP:GFP, were localized in unpaired cytoplasmic bodies containing ropelike, fibrillar structures. The paired bodies were similar to previously described viroplasms, while the unpaired bodies were similar to X-bodies. These data indicate that the accumulation of proteins expressed from different sgps of TMV has a specific spatial and temporal pattern in planta. In addition, the cytoplasmic bodies containing the $126 \mathrm{kDa}$ and $183 \mathrm{kDa}$ proteins are dynamic entities whose protein content and subcellular location change during infection.
\end{abstract}

Corresponding author: Roger N. Beachy; Telephone: 1-619-784-2550; Fax: 1-619-784-2994; E-mail: beachy@ scripps.edu

Current address of J. Szécsi, M. Bendahmane, R. N. Beachy: Donald Danforth Plant Science Center, Department of Biology, Box 1137, Washington University, St. Louis, MO 63130, U.S.A.

Judit Szécsi, Xin Shun Ding, and Chae Oh Lim contributed equally and should be considered co-first authors.
Traditional studies describing the development of infection sites of viruses in planta were hampered by the inability to identify the inoculated cells and to observe the invasion of adjacent cells during the first 36 to $48 \mathrm{~h}$ post inoculation (hpi). Moreover, it was not possible to study virus accumulation and gene expression in specific cells at precise locations within the infection site. The recent adaptation of the jellyfish (Aequorea victoria) green fluorescent protein (GFP) as an in vivo reporter of gene expression (Chalfie et al. 1994; Cody et al. 1993; Prasher et al. 1992) has greatly increased the ability to observe inter- and intracellular events accompanying virus infection by fluorescence or confocal microscopy (e.g., see Baulcombe et al. 1995; Heinlein et al. 1995, 1998; McLean et al. 1995; Oparka et al. 1995, 1997; Padgett et al. 1996; Santa Cruz et al. 1996).

To study the process of virus accumulation and local movement of the tobamoviruses, tobacco mosaic virus (TMV) and Obuda pepper vius (Ob or ObPV), GFP was fused to the viral movement protein (MP) and the MP:GFP fusion was expressed from the MP subgenomic promoter (sgp) during infection in protoplasts and in planta (Epel et al. 1996; Heinlein et al. 1995; Padgett et al. 1996). TMV MP is localized to plasmodesmata in virus-infected plants and in transgenic plants accumulating the MP (Atkins et al. 1991; Ding et al. 1992; Moore et al. 1992; Tomenius et al. 1987). Studies of the tobamovirus MP fused to the GFP revealed that the fusion protein accumulates in plasmodesmata, in fluorescent bodies, and associated with microtubules in infected plant cells. The size, shape, and number of the fluorescent bodies change throughout virus infection (Epel et al. 1996; Heinlein et al. 1998; Oparka et al. 1997; Padgett et al. 1996). Padgett et al. (1996) proposed that certain fluorescent bodies may be viroplasms or X-bodies, like those previously observed via electron microscopy in TMV-infected plants; these structures contain the $126 \mathrm{kDa}$ and $183 \mathrm{kDa}$ proteins (components of viral replicase) and were suggested to be sites of viral replication (Esau and Cronshaw 1967; Hills et al. 1987; Matthews 1981; Saito et al. 1987). The viral protein content of these fluorescent and nonfluorescent bodies and their subcellular location as the infection progresses have not been fully studied in planta.

In protoplasts, MP:GFP co-localizes with microtubules, cortical endoplasmic reticulum (ER), and punctate structures 
on or near the plasma membrane that may be cell wall adhesion sites (Heinlein et al. 1995, 1998). Furthermore, MP:GFP and $126 \mathrm{kDa}$ and $183 \mathrm{kDa}$ proteins appeared to co-localize on ER, particularly cortical ER (Heinlein et al. 1998). McLean et al. (1995) observed an interaction of MP with actin filaments as well as with microtubules. Based on these observations, it was proposed that MP is present in virus replication sites, and that the role of the MP includes binding to newly synthesized viral RNA and conducting it to the plasmodesmata with elements of the cytoskeleton and ER networks (Heinlein et al. 1995, 1998; McLean et al. 1995; Zambryski 1995).

In this study we report on the size and intensity of fluorescence from expanding infection sites in Nicotiana benthamiana produced by TMV mutants expressing MP:GFP driven by the MP sgp or free GFP driven by the coat protein (CP) sgp. We also describe the cell-specific pattern of accumulation of fluorescent proteins driven by two viral sgps at the leading edges of expanding infection sites. Lastly, the composition of cytoplasmic bodies induced by several of the mutants was analyzed throughout the infection sites and the relationship between these cytoplasmic bodies and the previously described X-bodies and viroplasms is defined.

\section{RESULTS}

Establishment and expansion of infection sites.

Five-week-old $N$. benthamiana plants were separately inoculated with transcripts of the virus constructs shown in Figure 1 , and the expanding infection sites were examined from 2.5 days post inoculation (dpi) by fluorescence microscopy. The plasmid pTMV-MP:GFP produces the $30 \mathrm{kDa}$ MP of TMV fused to the GFP under the regulation of the MP sgp. This construct lacks the CP gene. pTMV-MP:GFP-CP carries the $\mathrm{CP}$ gene under the regulation of the $\mathrm{CP}$ sgp, and is otherwise identical to pTMV-MP:GFP. In pTMV- $\Delta \mathrm{C}-\mathrm{GFP}$, the CP of TMV was replaced with the GFP. This construct expresses free GFP under the regulation of the CP sgp, and does not contain the $\mathrm{CP}$ gene.
Plants inoculated with transcripts of pTMV-MP:GFP or pTMV-MP:GFP-CP developed fluorescent disks that were visible at $2.5 \mathrm{dpi}$. At about $3 \mathrm{dpi}$, the disks developed to form rings consisting of 8 to 10 fluorescent upper epidermal and underlying mesophyll cells that encircled areas of very low fluorescence (e.g., 3.5 dpi; Fig. 2A and B). The rings continued to enlarge throughout the observation period (up to 1 week). Infections by the two viruses produced significantly different amounts of fluorescence reflecting the different amounts of MP:GFP produced. Infection with TMV-MP:GFP resulted in brighter fluorescent rings than infection with TMVMP:GFP-CP (compare Figure 2A and B; photomicrographs were taken with exposure times of $10 \mathrm{~s}$ and $60 \mathrm{~s}$, respectively). Based on these results, we estimate that the presence of the $\mathrm{CP}$ gene in the virus construct reduced the amount of MP by about 10- to 12-fold.

To determine whether the amount of the MP produced by TMV-MP:GFP or TMV-MP:GFP-CP is less than or similar to the amount of the MP produced by wild-type TMV during virus infection, tobacco protoplasts were inoculated separately with these constructs. Samples of infected protoplasts were taken at different hpi and the accumulation of the MP over time was compared by immunoblot assay. The results of these studies revealed that at each time point TMV-U1 produced greater amounts of MP than did TMV-MP:GFP or TMVMP:GFP-CP (Fig. 3). After comparing the intensity of protein bands on the immunoblot we concluded that wild-type TMVU1 produced at least three times and 20 times more MP than did TMV-MP:GFP and TMV-MP:GFP-CP, respectively. The blots were also reacted with antibody against the $126 \mathrm{kDa}$ and $183 \mathrm{kDa}$ replicase proteins of TMV and revealed that each virus produced approximately the same amount of these proteins at each time point, indicating that equal amounts of total proteins were loaded onto the gel at each time point (data not shown).

In inoculated leaves, infection by TMV- $\Delta$ C-GFP resulted in expanding fluorescent disks that were readily visible at $2.5 \mathrm{dpi}$ and remained as disks during the 2-week observation period

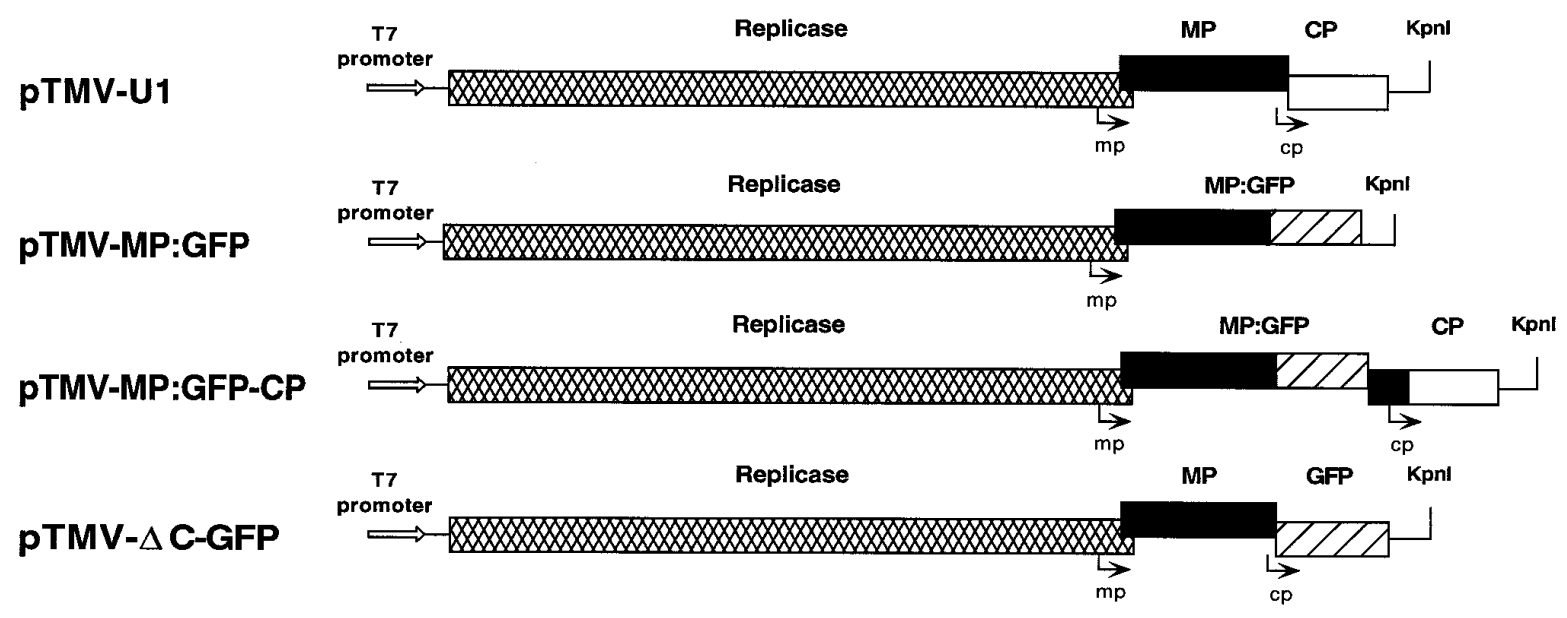

Fig. 1. Schematic diagram of tobacco mosaic virus (TMV) constructs used in this study. Modified forms of the TMV U3/12-4 full-length cDNA (referred to as TMV-U1; Holt and Beachy 1991) were constructed to express the green fluorescent protein (GFP) as a free protein from the coat protein (CP) subgenomic promoter (sgp) or as a fusion with viral movement protein (MP) from the MP sgp. Each cloned TMV cDNA is ligated to the T7 promoter; each construct was linearized with KpnI prior to transcription. Arrows represent sgps. Hatched pattern, replicase; black, MP; open box, CP; striped pattern, GFP; MP:GFP, fusion of MP and GFP. 
(e.g., 3.5 dpi; Fig. 2C). By comparing the fluorescence intensity of infection sites caused by the three different constructs, we estimated that disks produced by TMV- $\Delta \mathrm{C}$-GFP were two to three times brighter than the rings produced by TMVMP:GFP and 20 to 35 times brighter than infection sites produced by TMV-MP:GFP-CP.

Superimposing the fluorescent ring produced by TMVMP:GFP and the fluorescent disk produced by TMV- $\Delta \mathrm{C}-\mathrm{GFP}$, both at 3.5 dpi (Fig. 2A and $\mathrm{C}$, respectively), revealed a difference in size between the two infection sites (Fig. 2D). To compare the rate of expansion of infection sites, the diameters of 40 to 80 infection sites produced by each construct were determined at various time points between 2.5 and 5 dpi. The average size of infection sites is shown in Figure 4. At 2.5 dpi all infection sites were very small, and differed little from each other. From 3 dpi to the end of the observation period, the sizes of fluorescent rings caused by TMV-MP:GFP and TMV-MP:GFP-CP were not significantly different from each other. These results indicate that the rate of cell to cell spread of infection was similar for both viruses in spite of the different amounts of MP:GFP that accumulated (see Figure 3). At 3 $\mathrm{dpi}$, the fluorescent rings were significantly larger in diameter than disks caused by infections with virus that produced free GFP under the regulation of the CP sgp (i.e., TMV- $\Delta \mathrm{C}-\mathrm{GFP}$ ). These results indicated that gene expression from the MP sgp is regulated differently than that from the CP sgp.
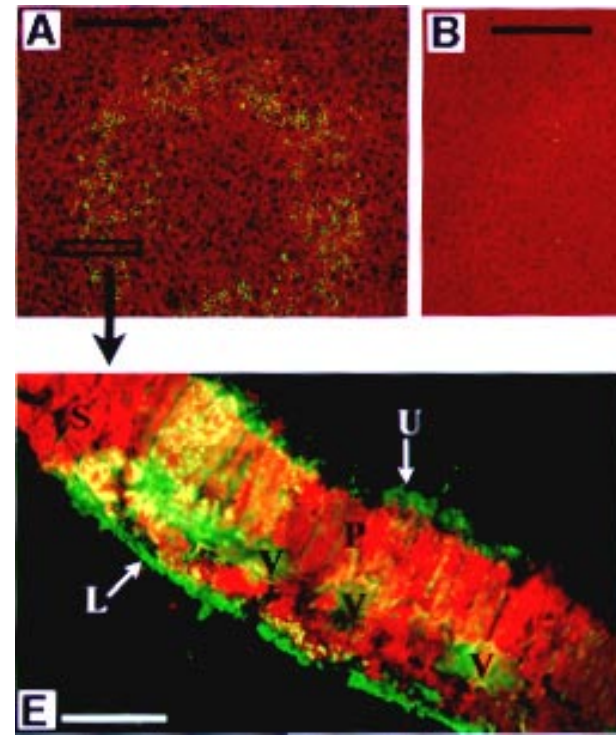
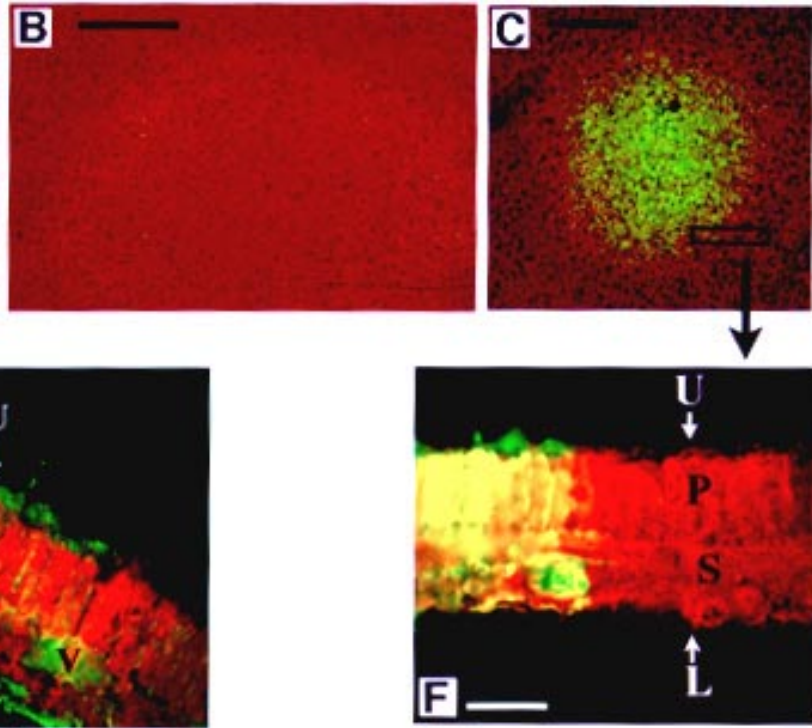

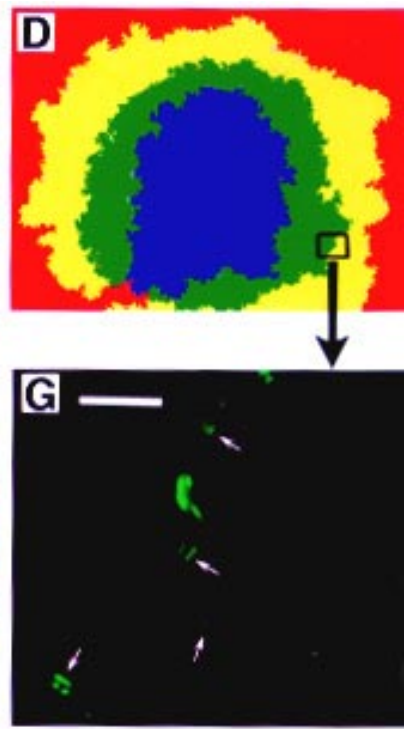

Fig. 2. Accumulation of green fluorescent protein (GFP) in Nicotiana benthamiana plant tissues infected with the different virus constructs. A-C, Infection sites at 3.5 days post infection (dpi). Bars represent $1 \mathrm{~mm}$. A, Fluorescent ring caused by TMV-MP:GFP infection. Fluorescence represents accumulation of MP:GFP fusion protein. Micrograph was taken with a $10 \mathrm{~s}$ exposure time. B, Fluorescent ring caused by TMV-MP:GFP-CP infection. Faint yellow color represents accumulation of MP:GFP fusion protein. Micrograph was taken with a 60 s exposure time. C, Fluorescent disk caused by TMV$\Delta \mathrm{C}$-GFP infection. Fluorescence represents accumulation of free GFP. Micrograph was taken with a $10 \mathrm{~s}$ exposure time. D, Overlay of fluorescent ring caused by TMV-MP:GFP and fluorescent disk caused by TMV- $\Delta$ C-GFP infection (A and $\mathbf{C}$, respectively) in pseudocolor. Yellow represents expression of MP:GFP; blue represents expression of free GFP. Green represents areas where expression of MP:GFP from the MP subgenomic promoter (sgp) and free GFP from the CP sgp overlap. E, Fresh tissue cross section of fluorescent ring caused by TMV-MP:GFP infection, taken from the boxed area in A. Fluorescence represents accumulation of MP:GFP. F, Fresh tissue cross section of fluorescent disk caused by TMV- $\Delta$ C-GFP infection, taken from the boxed area in C. Fluorescence represents accumulation of free GFP. E and F, Bar $=125 \mu \mathrm{m}$. U, upper epidermis; P, palisade parenchyma; S, spongy parenchyma; L, lower epidermis; V, vascular tissue. G, MP:GFP accumulation in cells infected with TMV-MP:GFP; magnification of boxed area in D representing paired fluorescent bodies. Arrows show paired bodies near the cell wall. Bar $=20 \mu \mathrm{m}$. TMV, tobacco mosaic virus; MP, movement protein; CP, coat protein.

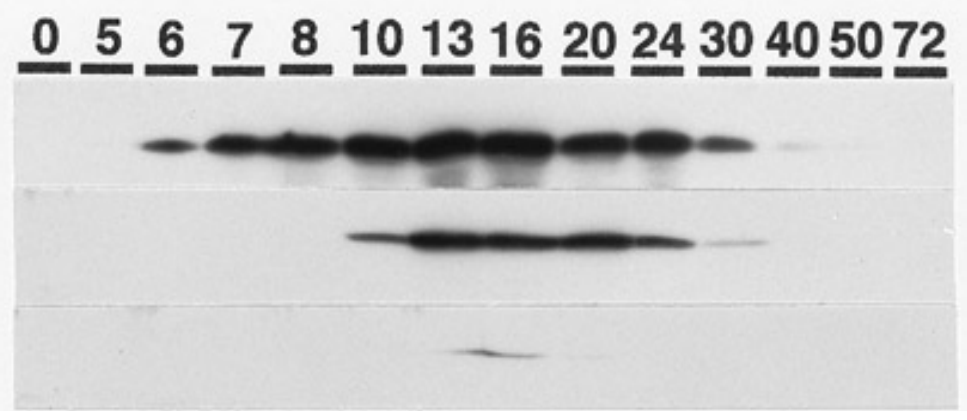

hpi

\section{TMV-U1}

\section{TMV-MP:GFP}

\section{TMV-MP:GFP-CP}

Fig. 3. Western blot (immunoblot) comparing the amount of movement protein (MP) produced by TMV-U1, TMV-MP:GFP, and TMV-MP:GFP-CP in infected protoplasts. Protoplast samples were harvested $0,5,6,7,8,10,13,16,20,24,30,40,50$, and $72 \mathrm{~h}$ post inoculation (hpi) and analyzed by immunoblot assay with antibody against the MP of tobacco mosaic virus (TMV). GFP, green fluorescent protein; CP, coat protein. 


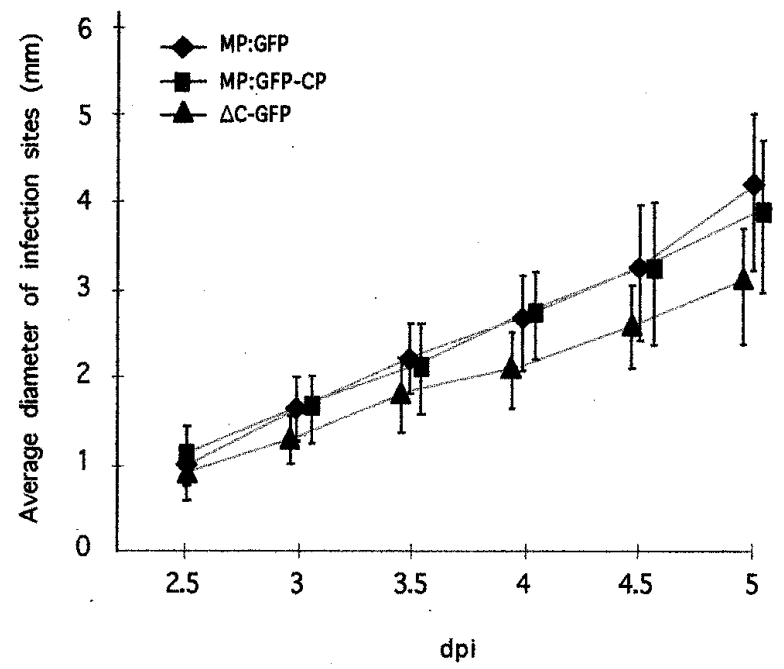

Fig. 4. Expansion of fluorescent rings and disks caused by three different virus constructs. Data points represent average diameters of 40 to 80 infection sites measured in two experiments at each time point with the standard deviations shown by vertical bars. Analysis of variance followed by least significant difference were used to determine significance of differences between mean values at each time point. Sizes of infection sites produced by TMV- $\Delta$ C-GFP were significantly different $(P<0.001)$ from those produced by the other virus constructs at 3.0 to 5.0 days post inoculation (dpi). TMV, tobacco mosaic virus; GFP, green fluorescent protein; MP, movement protein; $\mathrm{CP}$, coat protein.

\section{Cross-section analyses of infection sites.}

Cross sections of fresh tissues were taken through the infection sites caused by TMV-MP:GFP and TMV- $\Delta$ C-GFP in order to determine how each virus infection expands through leaf tissues. Tissue sections were observed by fluorescence microscopy (Fig. 2E and F) and the data are summarized in Figure 5.

Cross sections taken at different distances from the leading edge of the expanding fluorescent ring produced by TMVMP:GFP (Fig. 5A) revealed differences in the accumulation of fluorescence in different cell types. Near the leading edge of the infection site all cell types (i.e., epidermis, palisade, and spongy parenchyma) contained fluorescence (Fig. 5B-1), while farther into the infection site fluorescence is progressively lost from palisade and spongy parenchymal cells (Fig. 5B-2). Farther inward, fluorescence is lost from the upper epidermis (Fig. 5B-3), and lastly is lost from the lower epidermis (Fig. 5B-4). This creates the appearance of a fluorescent ring when viewed from the upper or lower epidermis (Fig. 2A). Furthermore, fluorescence was generally observed in epidermal cells in advance of fluorescence in the spongy and palisade parenchyma, resulting in a "concave" fluorescent leading edge of most rings (Fig. 2E).

A similar study was conducted on infection sites induced by TMV- $\Delta$ C-GFP; in such sites fluorescence was observed in all cell types throughout the infection site (Fig. 5C), resulting in the appearance of a fluorescent disk when viewed from the
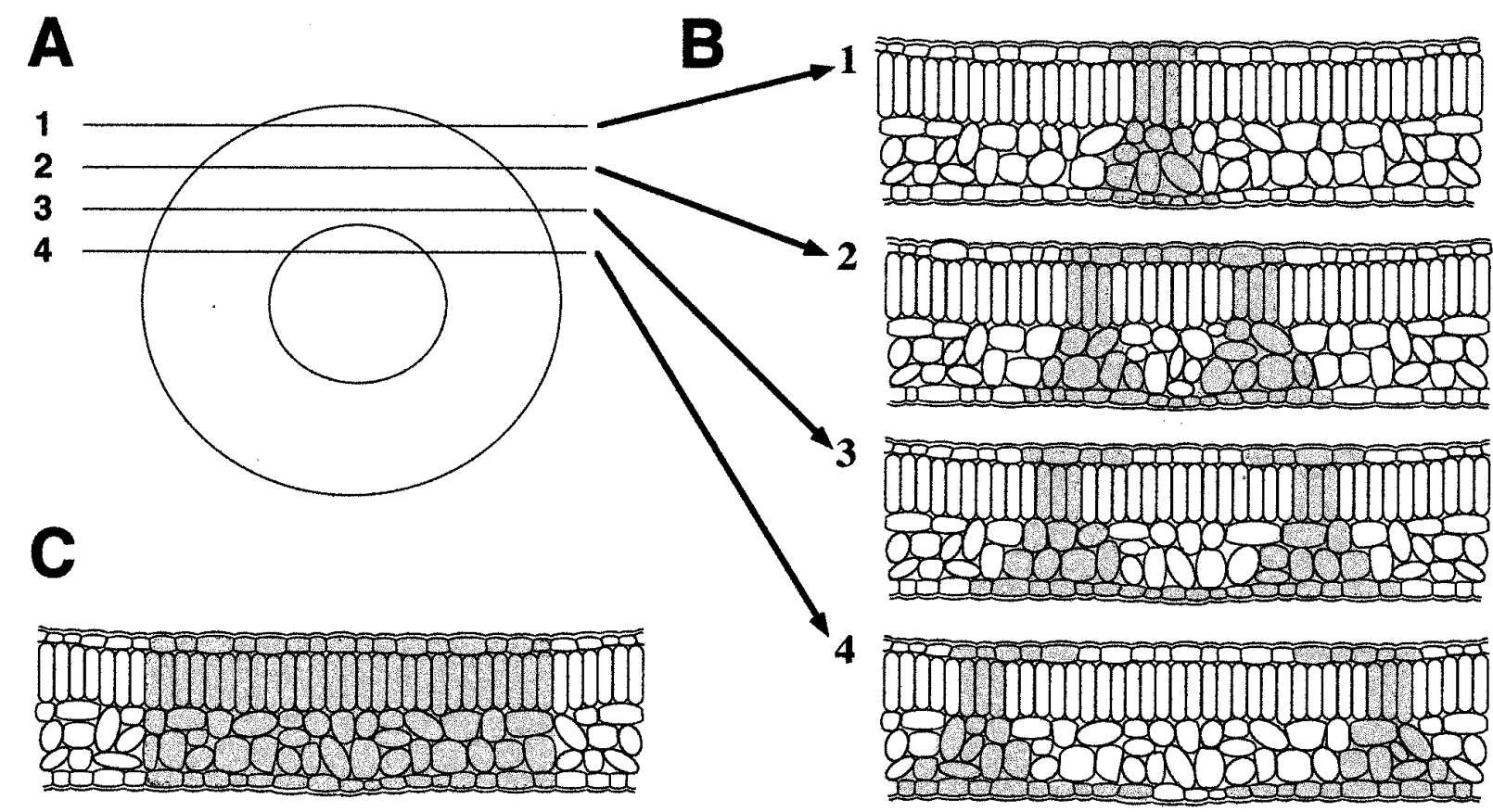

Fig. 5. Graphic representation of cross sections of fluorescent infection sites. A, Diagram of a fluorescent ring caused by TMV-MP:GFP. Lines show area from which cross sections were taken. B, Cross section of infection sites resulting from infection by TMV-MP:GFP. Shaded areas represent cells containing fluorescence due to MP:GFP accumulation. (1) Near leading edge of the expanding fluorescent ring all cell types accumulate MP:GFP, but accumulation is more advanced in the lower epidermis than in mesophyll cells, resulting in a "concave" shape of the leading edge. (2) In sections inward toward center of the fluorescent ring, GFP is absent from the palisade parenchyma and from the spongy parenchyma in the center of fluorescent ring. (3) Toward the faintly fluorescent center of the fluorescent ring GFP is absent from all cell types except lower epidermis. (4) Sections taken in middle of infection site show that in the center of the fluorescent ring most of the GFP is lost from the lower epidermis. C, Cross section of fluorescent disk caused by TMV- $\Delta$ C-GFP in the middle of the infection site. Shaded area represents free GFP accumulation in infected tissues. TMV, tobacco mosaic virus; MP, movement protein; GFP, green fluorescent protein. 
upper or lower epidermis (Fig. 2C). Furthermore, the "leading edge" of the fluorescent disks were essentially "blunt" in shape (Figs. 2F and 5C), i.e., they were not concave in appearance as described above (Fig. 2E). These observations support the hypothesis that expression from the CP sgp is regulated differently than expression from the MP sgp.

\section{Subcellular localization of viral proteins by fluorescence microscopy and immunocytochemistry.}

Previous studies from our lab described the accumulation of MP:GFP in different fluorescent structures observed in epidermal cells of $N$. benthamiana infected with ObM:Gfus (i.e., Ob MP fused to the GFP; Padgett et al. 1996) and in N. tabacum infected with TMV-MP:GFP (Heinlein et al. 1998). However, these studies did not investigate the accumulation of MP:GFP across the infection site or in comparison with accumulation of other viral proteins. In the present work we used immunocytochemistry to determine the location of MP:GFP and the TMV $126 \mathrm{kDa}$ and $183 \mathrm{kDa}$ proteins through the infection sites.

In $N$. benthamiana leaves infected by TMV-MP:GFP, epidermal cells located one to two cells behind the fluorescent leading edge of the infection site contained fluorescent paired bodies adjacent to the cell wall (Fig. 2G). Infection by TMVMP:GFP-CP also resulted in paired bodies, although at much lower frequency than in infection sites induced by TMVMP:GFP (data not shown). This was anticipated based on the results from the immunoblot assay showing that infection by TMV-MP:GFP-CP produced very low amounts of MP:GFP compared with TMV-MP:GFP (Fig. 3). When semi-thin (2 $\mu \mathrm{m})$ sections from leaves of $N$. benthamiana infected with TMV-MP:GFP were probed with an antibody against GFP and analyzed by light microscopy, a zone of four to six adjacent epidermal, mesophyll, and bundle sheath cells contained labeled cytoplasmic bodies (Fig. 6A). Cells in which antibody reactions were observed corresponded with the location of cells containing the green fluorescence in rings caused by TMV-MP:GFP (Fig. 2E). The labeled cytoplasmic bodies were generally smaller in size than chloroplasts (Fig. 6A; compare the size of the immunolabeled cytoplasmic bodies [arrowheads] and the toluidine blue-stained chloroplasts within the inset). The number of labeled bodies per cell section in this area ranged from one to seven with an average of 2.6 bodies per cell section. Most of the labeled bodies were paired on opposite sides of adjacent cell walls (70\% of 84 bodies counted in 30 cells). It is likely that these bodies represent the paired fluorescent bodies described above (Fig. 2G). When semi-thin $(2 \mu \mathrm{m})$ sections of the infection sites caused by TMV-MP:GFP-CP were probed with an antibody against GFP-labeled cytoplasmic bodies, similar in appearance to those induced by TMV-MP:GFP, were observed in all cell types within the fluorescent part of the ring (Fig. 7). However, fewer labeled bodies were observed in tissues infected with TMV-MP:GFP-CP compared with the number of labeled bodies observed after infection with TMV-MP:GFP. On average, 1.6 labeled bodies per cell section were found, and $11 \%$ of the labeled bodies were paired. These results are in agreement with the results of fluorescent microscopy described above, and correlate well with the amount of MP:GFP produced by these constructs (Fig. 3). Near the front of the infection sites caused by TMV-MP:GFP or TMV-MP:GFP-CP, in cells that contained paired bodies, cell walls of adjacent cells appeared to be closely appressed to each other (Figs. 6A and 7).

In the center of the infection sites, bodies were not paired and very few cytoplasmic bodies contained MP:GFP (less than one body per five cells). No labeled paired or unpaired bodies were observed in advance of the leading edge of the fluorescent rings (data not shown). We did not observe antibody labeling in control leaf sections infected with TMVMP:GFP that were probed with a polyclonal antibody against the TMV CP (TMV-MP:GFP lacks the CP gene), or in leaf sections from mock-inoculated plants probed with antibody against GFP (data not shown).

To further examine the contents of TMV-induced cytoplasmic bodies, thin serial sections prepared from tissue blocks previously studied by immunocytochemistry and light microscopy were analyzed by immunogold labeling and electron microscopy. In the center of the fluorescent ring, i.e., in cells abutting the region containing high fluorescence, unpaired cytoplasmic bodies containing electron-dense, ropelike structures were observed (Fig. 6B and $\mathrm{C}$, and data not shown). These bodies were labeled with antibody against the $126 \mathrm{kDa}$ and $183 \mathrm{kDa}$ proteins, but not with antibody against the GFP (Fig. 6B and C, respectively). Within the fluorescent ring, the paired electron- dense bodies, representing the black bodies observed by light microscopy (Fig. 6A), were labeled with antibodies against the $126 \mathrm{kDa}$ and $183 \mathrm{kDa}$ proteins, the MP, and the GFP (Fig. 6D and E, and Table 1). These paired bodies did not contain distinct ropelike structures and they were often connected by plasmodesmata (e.g., Fig. 6D and E). In all cells examined in this study, MP, GFP and the $126 \mathrm{kDa}$ and $183 \mathrm{kDa}$ replicase proteins accumulated only in virus-induced paired bodies, and were not detected in other parts of the cell (Table 1).

Thin sections prepared from tissues of mock-inoculated plants did not bind antibodies against the $126 \mathrm{kDa}$ and 183 $\mathrm{kDa}$ proteins, MP, or GFP (data not shown) and there was no immunogold labeling in sections from tissues infected with TMV-MP:GFP after incubation with anti-CP antibody (Fig. 6F and Table 1).

\section{DISCUSSION}

\section{Protein expression from the MP sgp and CP sgp is differentially regulated during TMV infection.}

In the present study we described the differences in accumulation of viral proteins between infection sites caused by different constructs of TMV that express MP:GFP fusion from the MP sgp or free GFP from the CP sgp in inoculated leaves of $N$. benthamiana.

Previously, Padgett et al. (1996) reported that infection of $N$. benthamiana with ObM:Gfus (which produces a fusion between the MP of $\mathrm{Ob}$ and GFP) produced fluorescent rings, while unfused GFP expressed from the CP sgp resulted in fluorescent disks. However, that study did not compare the sizes of infection sites resulting from infection with the two different constructs. In the present study, we determined that fluorescent rings resulting from infection with TMV constructs expressing MP:GFP from the MP sgp were significantly larger in diameter than fluorescent disks resulting from expression of GFP from the CP sgp on infected N. benthami- 
ana (Figs. 2D and 4). The differences in size of fluorescent sites can be explained by differences in the timing of gene expression from the two subgenomic promoters; i.e., the expression from the MP sgp precedes expression from the CP sgp during TMV infection. This is in agreement with results of previous studies in protoplasts, which showed that expression from the MP and the CP sgps is differentially regulated (Padgett et al. 1996; Watanabe et al. 1984). The sizes of fluo- rescent rings resulting from infection with TMV-MP:GFP and TMV-MP:GFP-CP were similar, indicating that the absence or presence of $\mathrm{CP}$ in the virus genome does not affect the rate of local spread of the infection.

In addition to differences in the timing of expression, there was also a difference in the amount of protein produced from the MP and CP sgps. The intensity of fluorescence in disks caused by TMV- $\Delta$ C-GFP was two to three times greater than

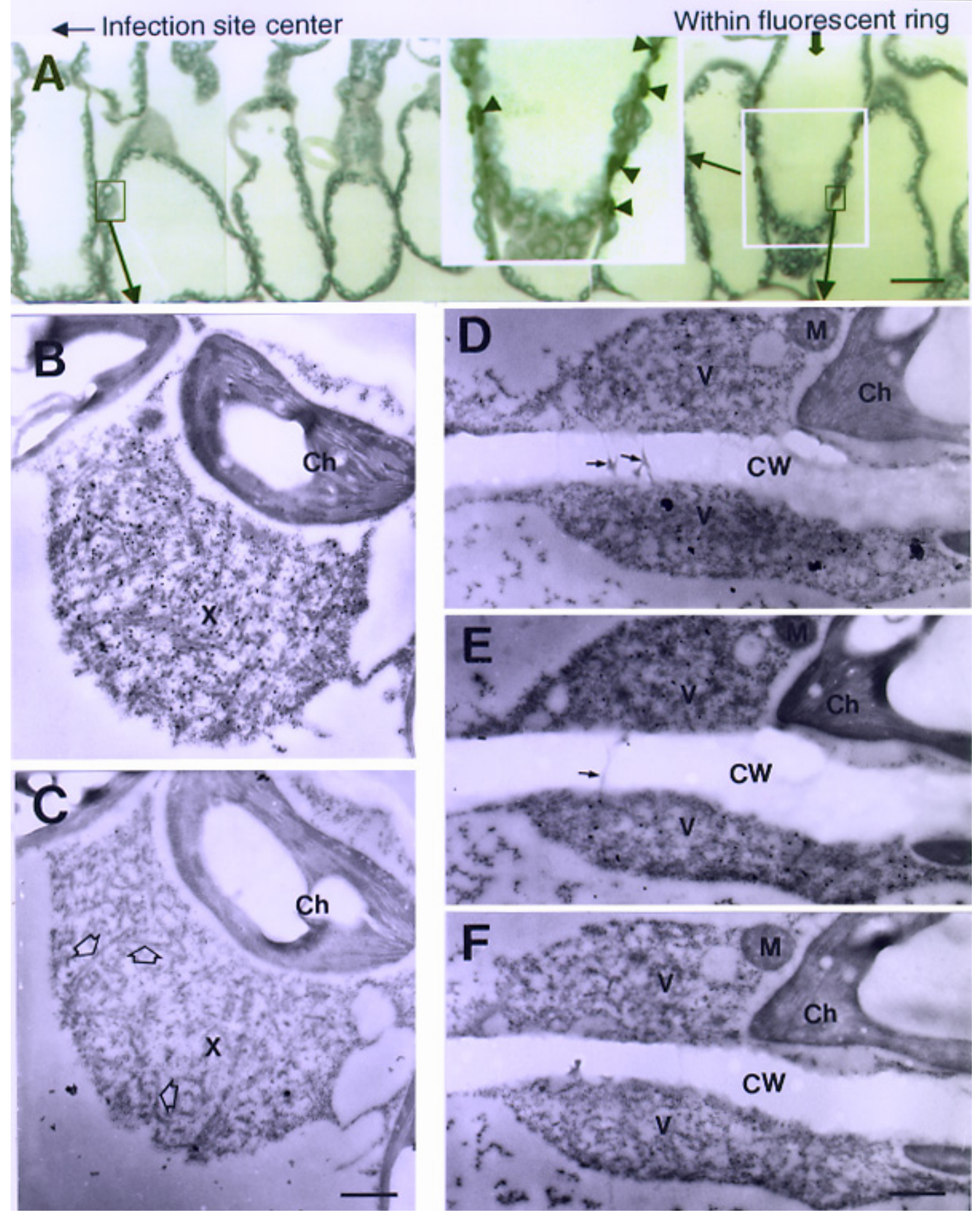


in rings produced by TMV-MP:GFP (compare Figure 2C with A). This is in agreement with results from previous studies in infected tobacco protoplasts that showed that expression from the CP sgp is substantially greater than that from the MP sgp (Epel et al. 1996; Padgett et al. 1996). Moreover, the amount of MP:GFP produced was correlated with the position of the gene relative to the $3^{\prime}$ end of the TMV-RNA. Plants infected with TMV-MP:GFP, which lacks the CP, accumulated higher levels of fluorescence than plants infected with TMV-MP:GFP-CP (compare Figure 2A and B). The differences in the intensity of fluorescence presumably reflect differences in expression of the gene and not differences in protein stability since the fusion proteins were identical for the two constructs (see Figure 1). In infected protoplasts it was also demonstrated that the amount of MP produced by these two constructs is less than the amount produced by the wild-type TMV-U1 virus (Fig. 3). Culver et al. (1993) previously reported that the amount of MP produced by TMV was affected by the position of the MP gene relative to the $3^{\prime}$ end in the virus genome. Increasing the amount of MP by removing the $\mathrm{CP}$ from the viral genome did not increase the local spread of the infection (Fig. 4). This is consistent with the report of Arce-Johnson et al. (1995), who concluded, based on studies of transgenic plants accumulating different amounts of MP, that the amount of MP required for local spread of TMV is much less than the amount of MP produced during TMV infection.
Analyses of cross sections taken through the infection sites caused by TMV-MP:GFP or TMV- $\Delta$ C-GFP revealed differences in the spatial patterns of fluorescence in infected $N$. benthamiana. Infection by TMV-MP:GFP produced fluorescent rings as a result of the loss (degradation) of MP:GFP in the middle of the infection site. Moreover, the accumulation and/or degradation of MP:GFP differs in different cell types; this resulted in a concave shape of the leading and trailing edge of the fluorescent ring (Figs. 2E and 5B). We considered the possibility that MP:GFP is transported from cells in which virus replication and MP:GFP production occur to immediately adjacent cells. It is known that there can be differences in the density of plasmodesmata between different leaf cell types (see review by Nelson and van Bel 1998). If the transport of MP from cells with active virus replication can be influenced by the density of plasmodesmata between different cell types this may result in asymmetrical distribution of MP:GFP at the leading edge of the infection site. However, it is also possible that nonfluorescent cells at the leading edge are engaged in virus replication (and synthesis of MP:GFP) that is not detected by the methods used in this study.

In contrast, cross sections of infection sites caused by TMV- $\Delta$ C-GFP revealed a "blunt" leading edge of the fluorescent rings (Fig. 2F), suggesting that production and accumulation of GFP from the CP sgp are concurrent, and are not influenced by the number or type of plasmodesmata between different cell types.

Fig. 6. Immunodetection of tobacco mosaic virus (TMV) $126 \mathrm{kDa}$ and $183 \mathrm{kDa}$ proteins and MP:GFP fusion protein in serial leaf sections prepared from a mature leaf of Nicotiana benthamiana infected with TMV-MP:GFP. A, After reaction with anti-GFP antibody and silver enhancement, section was stained with toluidine blue and photographed with a light microscope. Signal appears as black areas in the cytoplasm adjacent to the cell wall. Left side of section is in the center of infection site; right side is near leading edge of the fluorescent ring. Inset: Arrowheads indicate location of paired bodies in adjacent, infected cells. Bar $=8 \mu \mathrm{m}$. B and $\mathbf{C}$, Micrographs of immunogold-labeled serial sections taken from black-boxed area on left side of A. B, Section labeled with an antibody against the TMV $126 \mathrm{kDa}$ and $183 \mathrm{kDa}$ proteins showing deposits of immunogold within an unpaired cytoplasmic body (X-body). C, Section probed with antibody against GFP. Open arrows indicate location of tubular or "ropelike" structures within unpaired body. Micrographs are rotated clockwise approximately $100^{\circ}$ in relation to section shown in A. Bar $=0.3 \mu \mathrm{m}$. Ch, chloroplast; X, X-body. D-F, Micrographs of immunogold-labeled serial sections taken from black-boxed area on right side of A. D and E, Sections labeled with antibody against the TMV 126 $\mathrm{kDa}$ and $183 \mathrm{kDa}$ proteins and GFP, respectively, showing immunogold deposits within paired cytoplasmic bodies (viroplasms) on opposite sides of the cell wall. F, Section incubated with an antibody against the CP of TMV. Micrographs were rotated clockwise approximately $100^{\circ}$ in relation to section shown in A. Bar $=0.6 \mu \mathrm{m}$. Black arrows indicate presence of plasmodesmata between paired bodies. Ch, chloroplast; CW, cell wall; M, mitochondria; V, viroplasms. MP, movement protein; GFP, green fluorescent protein.

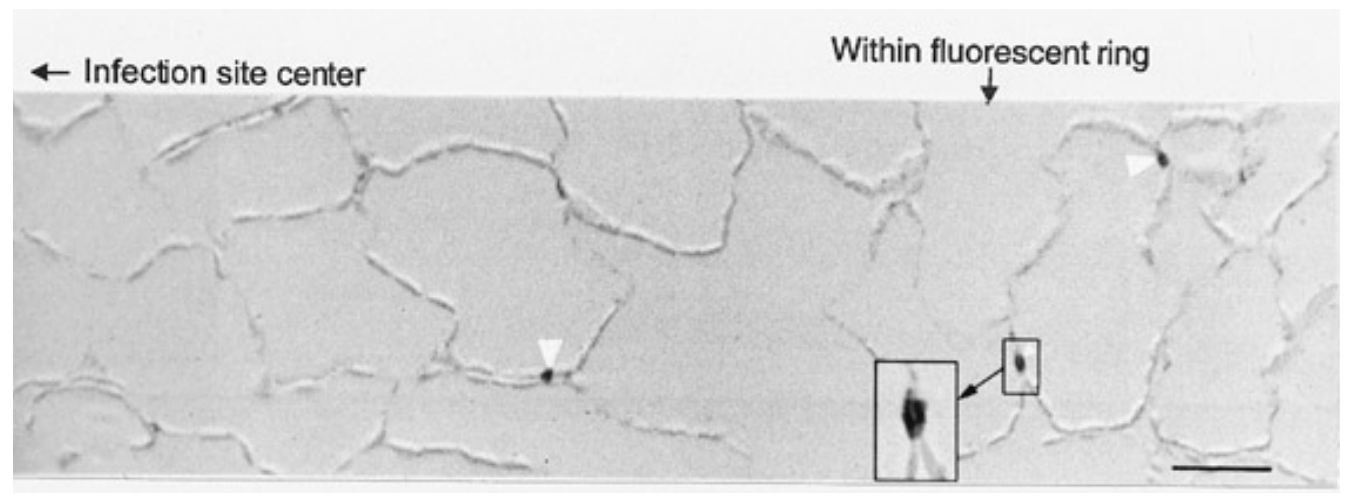

Fig. 7. Immunodetection of the MP:GFP fusion protein in a leaf section infected with TMV-MP:GFP-CP. After reaction with anti-GFP antibody and silver enhancement, section was photographed with a light microscope with Nomarski optics. Signals appear as black areas in the cy toplasms adjacent to the cell walls (arrowheads). Left side of section is in the center of the infection site; right side is near leading edge of the fluorescent ring. Inset: presence of a paired cytoplasmic body adjacent to cell walls of two infected cells. Bar $=10.6 \mu \mathrm{m}$. TMV, tobacco mosaic virus; MP, movement protein; GFP, green fluorescent protein; $\mathrm{CP}$, coat protein. 
The cytoplasmic bodies vary in their location and MP content as the infection progresses, but always contain the $126 \mathrm{kDa}$ and $183 \mathrm{kDa}$ proteins.

In the epidermal cells within the fluorescent ring caused by TMV-MP:GFP and TMV-MP:GFP-CP, fluorescent paired bodies were observed adjacent to cell walls (Fig. 2G). Since the amount of MP produced by wild-type TMV is greater than the amount produced by TMV-MP:GFP or TMV-MP:GFP-CP, we suggest that paired bodies exist in infection sites caused by TMV-U1. Using immunocytochemistry and light microscopy, we observed virus-induced cytoplasmic bodies throughout the infection site caused by TMV-MP:GFP and TMV-MP:GFP$\mathrm{CP}$; however, only cells within the fluorescent ring contained paired bodies (Figs. 6 and 7). The paired bodies were electron dense and similar in appearance to "viroplasms" described by Saito et al. (1987). In most instances, the paired bodies contained plasmodesmata between them when observed under the electron microscope (e.g., Fig. 6D and E). Immunogold labeling revealed that both MP:GFP and the $126 \mathrm{kDa}$ and 183 $\mathrm{kDa}$ proteins are present in the paired bodies (Fig. 6D and $\mathrm{E}$ ).

In contrast to findings within the fluorescent ring, in the faintly fluorescent center of the ring (i.e., in cells that represent a more mature area of virus accumulation) virus-induced bodies were not paired (Fig. 6A). These bodies accumulated the $126 \mathrm{kDa}$ and $183 \mathrm{kDa}$ proteins and contained distinct tubule or "ropelike" structures (Fig. 6B), and were similar in appearance to X-bodies (Hills et al. 1987). MP:GFP was absent from these structures (Fig. 6C). These data support the previous suggestion made by Saito et al. (1987) that viroplasms may be less mature forms of X-bodies. In addition, our results indicate that viroplasms contain MP, but X-bodies do not. It has been determined that paired bodies within the fluorescent ring are enriched in the microtubule-associated protein EF-1 $\alpha$ (X. S. Ding and R. S. Nelson, in preparation).

Recently, Oparka et al. (1997) demonstrated that plasmodesmata within the fluorescent ring exhibit increased size exclusion limits (SEL) but cells in the faintly fluorescent center of the ring do not, despite the presence of MP in their plasmodesmata. Those observations, in conjunction with the results presented here, support the hypothesis that increased SEL and intercellular spread of infection occur in cells with active MP synthesis. Moreover, the presence of MP within the paired fluorescent bodies suggests a role of the MP in establishing or maintaining these cytoplasmic structures. The pres-

Table 1. Subcellular location and quantity of immunogold particles in cells containing high levels of GFP fluorescence within the fluorescent ring produced by TMV-MP:GFP on Nicotiana benthamiana ${ }^{\mathrm{a}}$

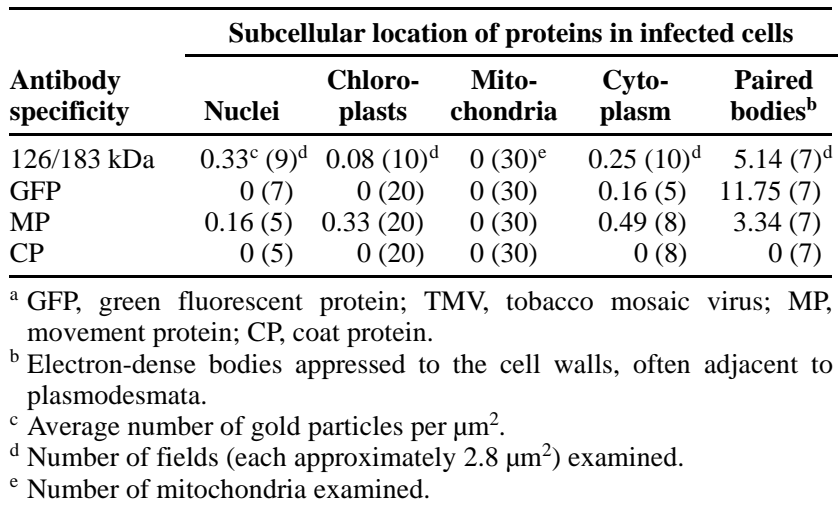

ence of the $126 \mathrm{kDa}$ and $183 \mathrm{kDa}$ proteins in these bodies implicates them as sites for RNA replication and raises the possibility that the spread of virus to adjacent cells is a consequence of the replication process. The RNA may be transported to adjacent cells as single-stranded RNA or as replication intermediate RNA in complex with MP as well as with replicase. Clearly, the paired bodies are complicated entities whose function in the replication of viral RNA and cell-to-cell spread of TMV requires further study.

\section{MATERIALS AND METHODS}

\section{DNA constructs.}

Wild-type and three different mutants of the TMV fulllength cDNA clone U3/12-4 (Holt and Beachy, 1991) were used in these experiments (Fig. 1). Wild-type TMV is also referred to as TMV-U1. The construct pTMV-MP:GFP contains the GFP fused with the MP (previously referred to as TMV-M:GfusBr; Heinlein et al. 1998); the GFP contained a threonine at position 65 in place of serine (S65T) to increase fluorescence (Heim et al. 1995). pTMV-MP:GFP-CP is similar to pTMV-MP:GFP but in addition encodes the CP of TMV (previously referred as TMV-MfCP; Heinlein et al. 1998). pTMV- $\Delta$ C-GFP produces the GFP as free protein from the CP sgp, and does not produce CP (Heinlein et al. 1995).

\section{In vitro transcription, and plant and protoplast inoculation.}

To produce infectious transcripts, $1 \mu \mathrm{g}$ of each plasmid was linearized with $K p n I$ and, after removal of the overhanging nucleotides by treatment with Klenow fragment of DNA polymerase $\mathrm{I}$, in vitro transcription reactions were conducted with T7 RNA Polymerase (Megascript T7 transcription kit; Ambion, Austin, TX). The upper surface of expanding leaves of 5-week-old $N$. benthamiana plants was mechanically inoculated as described (Holt and Beachy 1991). For immunocytochemistry, pTMV-MP:GFP was transcribed as described (Shintaku et al. 1996) and transcripts were mechanically applied to the fully expanded fifth leaf of 5-week-old $N$. benthamiana plants. Infected plants were maintained in a growth chamber at 23 to $24^{\circ} \mathrm{C}$ with $14 / 10 \mathrm{~h}$ or $16 / 8 \mathrm{~h}$ (for immunocytochemistry) light/dark photoperiods.

Protoplasts of BY2 tobacco cell line were prepared and electroporated with transcripts as described (Watanabe et al. 1982; Okada et al. 1986). Approximately $0.5 \times 10^{4}$ infected protoplasts were harvested at $0,5,6,7,8,10,13,16,20,24$, $30,40,50$, and $72 \mathrm{hpi}$ and half of each sample was used for immunoblot analysis as described (Sambrook et al. 1989). Membranes were blocked in Tris-buffered saline buffer and $0.05 \%$ Tween 20 (TBST) containing 4\% nonfat dry milk, followed by incubation with antibodies against MP (Deom et al. 1987 ) or the $126 \mathrm{kDa}$ and $183 \mathrm{kDa}$ proteins (Nelson et al. 1993) diluted 1:1,000 in TBST. After incubation in the first antibody, the membranes were washed and a horseradishperoxidase tagged secondary antibody (Pierce, Rockford, IL) diluted 1:50,000 in TBST was added. The blots were developed with the SuperSignal Chemiluminescent Substrate (Pierce) as described, followed by exposure of films for $0.5,1$, 10 , or $30 \mathrm{~min}$. The intensity of the protein bands was measured with the Multi-Analyst computer program from Bio-Rad (Hercules, CA). 


\section{Analysis of infection sites.}

Infection sites were observed at various times after inoculation with a Nikon (Melville, NY) Optiphot 2UD microscope with epi-fluorescence attachment EFD-3 with the Nikon B-2A filter cube containing a 470 to $490 \mathrm{~nm}$ excitation filter, a DM505 nm dichroic mirror, and a BA520 nm barrier filter. For analysis of expansion of infection sites, pieces from the infected leaves were removed at each time point (from 2.5 to 5 dpi) and the diameter of each infection site was recorded. The experiments were performed twice and data were combined. For analysis of cross sections of infection sites, fluorescent rings and disks were excised with a razor blade and immediately embedded in a liquid mixture $\left(30^{\circ} \mathrm{C}\right)$ of $3 \%$ low-meltingtemperature agarose (Sigma, St. Louis, MO) and 2\% agarose (Fisher, Pittsburgh, PA). After solidification of the blocks, $100-\mu \mathrm{m}$ leaf sections were prepared with a Vibrotome and samples were collected on microscope slides. Slides were mounted in $50 \%$ glycerol, $5 \mathrm{mM}$ EGTA in phosphate-buffered saline (PBS) buffer.

Photographs were taken with Kodak (Rochester, NY) Ectachrome 400 (Fig. 2A-C, E, and F) or Kodak EPH p1600 (Fig. 2G) film. Original 35-mm slides were scanned and Adobe Photoshop (Adobe Systems, San Jose, CA) was used to assemble figures.

\section{Tissue fixation, embedding, and immunocytochemistry.}

At 5 dpi (for TMV-MP:GFP) or 4 dpi (for TMV-MP:GFP$\mathrm{CP})$, leaf pieces $(4 \times 6 \mathrm{~mm})$ containing a fluorescent infection site were excised from inoculated leaves of $N$. benthamiana. Each sampled tissue extended from the center of an infection site to beyond the fluorescent zone. Leaf pieces from mockinoculated leaves were harvested and used as controls. All leaf pieces were fixed and embedded in LR White resin as previously described (Ding et al. 1996b, and references therein).

Semi-thin sections $(2 \mu \mathrm{m})$ prepared from the embedded leaf tissues were analyzed for accumulation of MP:GFP or CP by a double-sided labeling technique (Ding et al. 1996a). IgG against GFP (Clontech Laboratories, Palo Alto, CA) or CP (Ding et al. 1996b) was diluted to 1:250 and 1:1,000 (vol/vol), respectively, in an antibody dilution buffer $(0.1 \mathrm{M}$ phosphate buffer, $\mathrm{pH} 7.0$ containing $2 \%$ [wt/vol] bovine serum albumin and $0.01 \%$ [vol/vol] Tween 20). Sections with adequate labeling were identified by observing signal under a light microscope and thin serial sections $(100 \mathrm{~nm})$ from these areas were obtained for further analysis at the subcellular level under an electron microscope as described (Ding et al. 1996b). Antibodies against the $126 \mathrm{kDa}$ and $183 \mathrm{kDa}$ proteins (Nelson et al. 1993), MP (Deom et al. 1987), CP, and GFP were diluted to $1: 8,000,1: 800,1: 10,000$, and $1: 2,500$ ( $\mathrm{vol} / \mathrm{vol})$, respectively, in antibody dilution buffer. After post-staining with uranyl acetate and lead citrate, sections were examined and photographed as described (Ding et al. 1995).

\section{ACKNOWLEDGMENTS}

We thank Christophe Reichel, Ted Kahn, Michael Frese, and Manfred Heinlein for helpful comments during the preparation of the manuscript. We thank Hal Padgett for antibody against the $126 \mathrm{kDa}$ and $183 \mathrm{kDa}$ proteins. We are grateful to Sally Jo Leitner for growth and maintenance of plants. This work was supported by grants to R. N. B. from the National Science Foundation (MCB-9631124) and The Binational US-Israel BARD program (IS-2636-95), and by a grant to the PMBBRC-TSRI joint laboratory from the Korea Science and Engineering Foundation.

\section{LITERATURE CITED}

Arce-Johnson, P., Kahn, T. W., Reimann-Philipp, U., RiveraBustamante, R., and Beachy, R. N. 1995. The amount of movement protein produced in transgenic plants influences the establishment, local movement, and systemic spread of infection by movement proteindeficient tobacco mosaic virus. Mol. Plant-Microbe Interact. 8:415423.

Atkins, D., Hull, R., Wells, B., Roberts, K., Moore, P., and Beachy R. N. 1991. The tobacco mosaic virus $30 \mathrm{~K}$ movement protein in transgenic tobacco plants in located to plasmodesmata. J. Gen. Virol. 72:209211

Baulcombe, D. C., Chapman, S., and Santa Cruz, S. 1995. Jellyfish green fluorescent protein as a reporter for virus infection. Plant J. 7: 1045-1053.

Chalfie, M., Tu, Y., Euskirchen, G., Ward, W. W., and Prasher, D. C. 1994. Green fluorescent protein as a marker for gene expression. Science 263:802-805.

Cody, C. W., Prasher, D. C., Westler, W. M., Prendergast, F. G., and Ward, W. W. 1993. Chemical structure of the hexapeptide chromophore of the Aequorea green-fluorescent protein. Biochemistry 32: 1212-1218.

Culver, J. N., Lehto, K., Close, S. M., Hilf, M. E., and Dawson, W. O. 1993. Genomic position affects the expression of tobacco mosaic virus movement and coat protein genes. Proc. Natl. Acad. Sci. USA 90: 2055-2059.

Deom, C. M., Oliver, M. J., and Beachy, R. N. 1987. The 30-kilodalton movement protein of tobacco mosaic virus potentiates virus movement. Science 237:389-394.

Ding, B., Haudenshield, J. S., Hull, R., Wolf, S., Beachy, R. N., and Lucas, W. J. 1992. Secondary plasmodesmata are specific sites of localization of tobacco mosaic virus movement protein in transgenic tobacco plants. Plant Cell 4:915-128.

Ding, X. S., Carter, S. A., and Nelson, R. S. 1996a. Enhanced cytochemical detection of viral proteins and RNAs using double-sided labeling and light microscopy. Biotechniques 20:111-115.

Ding, X. S., Shintaku, M. H., Arnold, S. A., and Nelson, R. S. 1995. Accumulation of mild and severe strains of tobacco mosaic virus in minor veins of tobacco. Mol. Plant-Microbe Interact. 8:32-40.

Ding, X. S., Shintaku, M. H., Carter, S. A., and Nelson, R. S. 1996b. Invasion of minor veins of tobacco leaves inoculated with tobacco mosaic virus mutants defective in phloem-dependent movement. Proc. Natl. Acad. Sci. USA 93:11155-11160.

Epel, B. L., Padgett, H. S., Heinlein, M., and Beachy, R. N. 1996. Plant virus movement protein dynamics probed with a GFP-protein fusion. Gene 173:75-79.

Esau, K., and Cronshaw, J. 1967. Relation of tobacco mosaic virus to the host cells. J. Cell Biol. 33:665-678.

Heim, R., Cubitt, A. B., and Tsien, R. Y. 1995. Improved green fluorescence. Nature 373:663-664.

Heinlein, M., Epel, B. L., Padgett, H. S., and Beachy, R. N. 1995. Interaction of tobamovirus movement protein with the plant cytoskeleton. Science 270:1983-1985.

Heinlein, M., Padgett, H. S., Gens, J. S., Pickard, B. G., Casper, S. J., Epel, B. L., and Beachy, R. N. 1998. Changing patterns of localization of tobacco mosaic virus movement protein and replicase to endoplasmic reticulum and microtubules during infection. Plant Cell 10: 1107-1120.

Hills, G. J., Plaskitt, K. A., Young, N. D., Dunigan, D. D., Watts, J. W., Wilson, T. M. A., and Zaitlin, M. 1987. Immunogold localization of the intracellular sites of structural and non structural tobacco mosaic virus proteins. Virology 161:488-496.

Holt, C. A., and Beachy, R. N. 1991. In vivo complementation of infectious transcripts from mutant tobacco mosaic virus cDNAs in transgenic plants. Virology 181:109-117.

Matthews, R. E. F. 1981. Plant Virology. 2nd ed. Academic Press, New York.

McLean, B. G., Zupan, J., and Zambryski, P. C. 1995. Tobacco mosaic virus movement protein associates with the cytoskeleton in tobacco cells. Plant Cell 7:2101-2114. 
Moore, P. J., Fenczik, C. A., Deom, C. M., and Beachy, R. N. 1992. Developmental changes in plasmodesmata in transgenic tobacco expressing the movement protein of tobacco mosaic virus. Protoplasma 170:115-127.

Nelson, R. S., Li, G., Hodgson, R. A. J., Beachy, R. N., and Shintaku, M. H. 1993. Impeded phloem-dependent accumulation of the masked strain of tobacco mosaic virus. Mol. Plant-Microbe Interact. 6:45-54.

Nelson, R. S., and van Bel, A. J. E. 1998. The mystery of virus trafficking into, through and out of vascular tissue. Prog. Bot. 59:476-533.

Okada, K., Nagata, T., and Takebe, I. 1986. Introduction of functional RNA into protoplasts by electroporation. Plant Cell Physiol. 27:619626.

Oparka, K. J., Prior, D. A. M., Santa Cruz, S., Padgett, H. S., and Beachy, R. N. 1997. Gating of epidermal plasmodesmata is restricted to the leading edge of expanding infection site. Plant J. 12:781-789.

Oparka, K. J., Roberts, A. G., Prior, D. A. M., Chapman, S., Baulcombe, D. C., and Santa Cruz, S. 1995. Imaging the green fluorescent protein in plants - viruses carry the torch. Protoplasma 189:1367-1373.

Padgett, H. S., Epel, B. L., Kahn, T. W., Heinlein, M., Watanabe, Y., and Beachy, R. N. 1996. Distribution of tobamovirus movement protein in infected cells and implication for cell-to-cell spread of infection. Plant J. 10:1079-1088.

Prasher, D. C., Eckenrode, V. K., Ward, W. W., Prendergast, F. G., and Cormier, M. J. 1992. Primary structure of the Aequorea victoria green-fluorescent protein. Gene 111:229-233.

Saito, T., Hosokawa, D., Meshi, T., and Okada, Y. 1987. Immunocyto- chemical localization of the $130 \mathrm{~K}$ and $180 \mathrm{~K}$ (putative replicase component) of tobacco mosaic virus. Virology 160:477-481.

Sambrook, J., Fritsch, E. F., and Maniatis, T. A. 1989. Molecular Cloning: A Laboratory Manual. 2nd ed. Cold Spring Harbor Laboratory, Cold Spring Harbor, NY.

Santa Cruz, S., Chapman, S., Roberts, A. G., Roberts, I. M., Prior, D. A. M., and Oparka, K. J. 1996. Assembly and movement of a plant virus carrying a green fluorescent protein overcoat. Proc. Natl. Acad. Sci. USA 93:6286-6290.

Shintaku, M. H., Carter, S. A., Bao, Y., and Nelson, R. S. 1996. Mapping nucleotides in the 126-kDa protein gene that control the differential symptoms induced by two strains of tobacco mosaic virus. Virology 221:218-225.

Tomenius, K., Clapham, D., and Meshi, T. 1987. Localization by immunogold cytochemistry of the virus-coded $30 \mathrm{~K}$ protein in plasmodesmata of leaves infected with tobacco mosaic virus. Virology 160:363371

Watanabe, Y., Emori, Y., Ooshika, I., Meshi, T., Ohno, T., and Okada, Y. 1984. Synthesis of TMV-specific RNAs and proteins at the early stage of infection in tobacco protoplasts: Transient expression of the $30 \mathrm{~K}$ protein and its RNA. Virology 133:18-24.

Watanabe, Y., Ohno, T., and Okada, Y. 1982. Virus multiplication in tobacco protoplast inoculated with tobacco mosaic virus RNA encapsulated in large unilamellar vesicle liposomes. Virology 120:478-480.

Zambryski, P. 1995. Plasmodesmata: Plant channels for molecules on the move. Science 270:1943-1944. 
Erratum, Volume 12 no. 2, 1999

Figure 6B-F, from the paper by Judit Szécsi, Xin Shun Ding, Chae Oh Lim, Mohammed Bendahmane, Moo Je Cho, Richard S. Nelson, and Roger N. Beachy (12:143-152) entitled "Development of Tobacco Mosaic Virus Infection Sites in Nicotiana benthamiana," has been reprinted here to better display the $20 \mathrm{~nm}$ gold particles over the X-body (X, panel $\mathbf{B}$ ) or viroplasms (V, panels $\mathbf{D}$ and $\mathbf{E}$ ) after labeling with antibody against the tobacco mosaic virus 126 and $183 \mathrm{kDa}$ proteins (panels $\mathbf{B}$ and $\mathbf{D}$ ) or green fluorescent protein (panel $\mathbf{E}$ ).
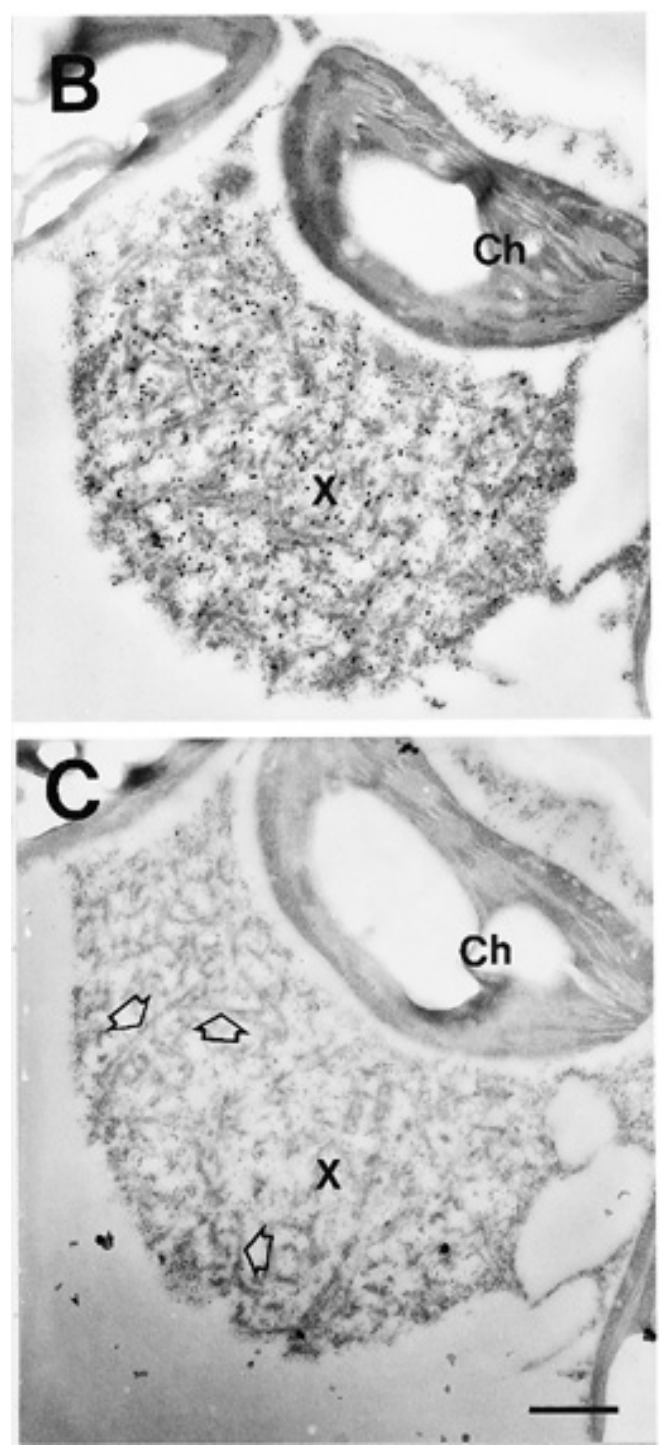
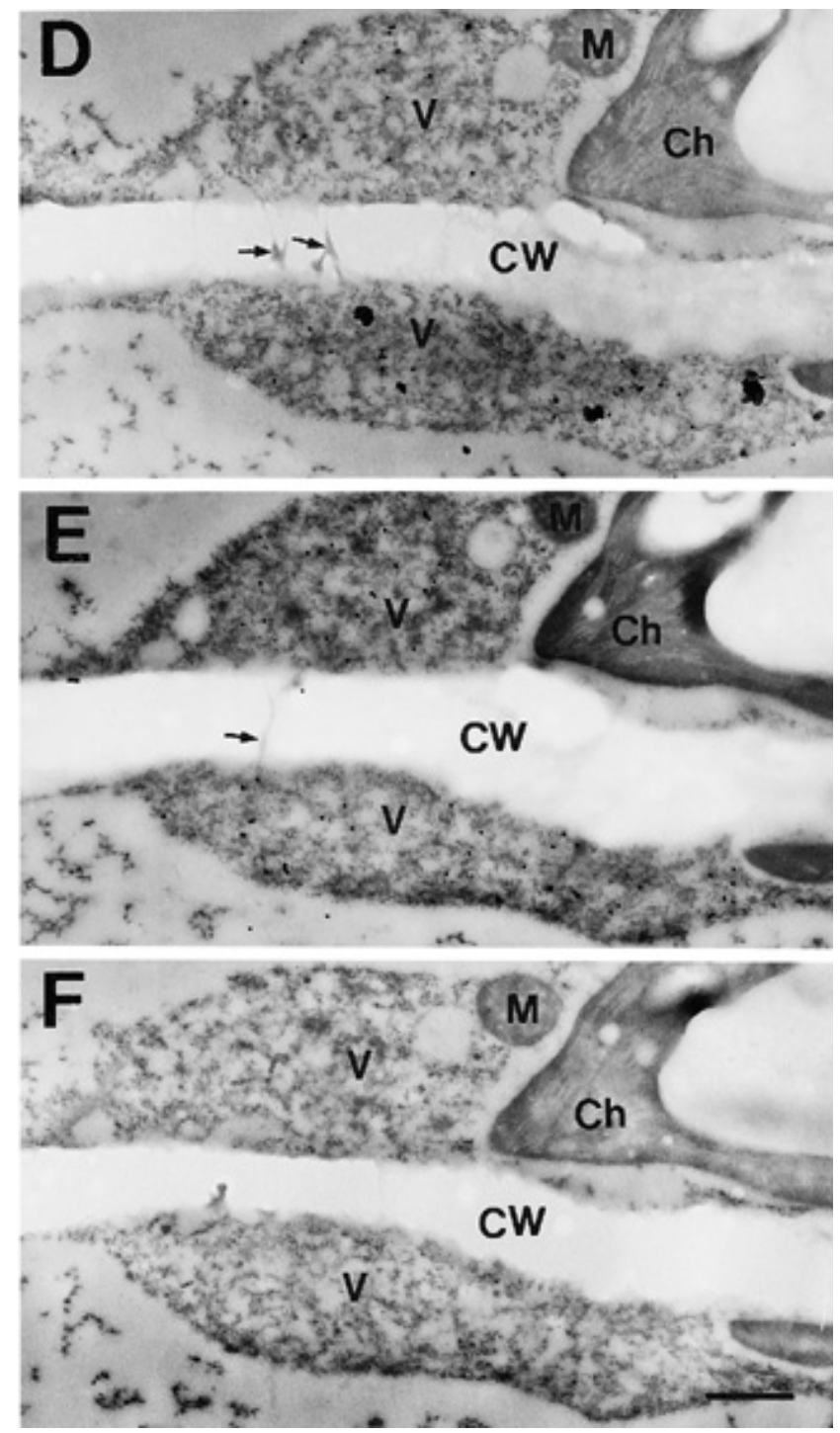